\title{
NOUVELLE
}

\section{Les vaisseaux sanguins HEV}

Des vaisseaux spécialisés

dans le recrutement des lymphocytes

Christine Moussion ${ }^{1,2}$, Jean-Philippe Girard ${ }^{1,2}$

${ }^{1}$ CNRS, institut de pharmacologie et de biologie structurale (IPBS), 205 route de Narbonne, 31077 Toulouse, France;

${ }^{2}$ Université Paul Sabatier de Toulouse, IPBS, 31077 Toulouse, France.

jean-philippe.girard@ipbs.fr
> En condition physiologique, les cellules sanguines empruntent la boucle circulatoire sans entrer en contact avec les tissus environnants, car l'endothélium constitue une barrière infranchissable entre ces deux territoires. La seule exception, qui sous-tend l'ensemble de la surveillance immunitaire chez les mammifères, est le passage massif de lymphocytes du sang vers les organes lymphoïdes secondaires. Les lymphocytes, cellules effectrices de la réponse immunitaire adaptative, patrouillent au sein de l'organisme à la recherche d'antigènes étrangers contre lesquels ils sont dirigés. Ils circulent continuellement du sang vers les organes lymphoïdes secondaires - dont les ganglions lymphatiques -, puis transitent dans le système lymphatique et regagnent la circulation sanguine via le canal thoracique. Les ganglions lymphatiques collectent l'ensemble des antigènes issus des tissus périphériques et constituent le site d'activation des lymphocytes en cas d'infection [1]. Le transit des lymphocytes naïfs du sang vers le tissu lymphoïde ganglionnaire nécessite une étape de migration transendothéliale, qui prend place spécifiquement au sein des veinules postcapillaires appelées HEV (high endothelial venule). Ces vaisseaux sanguins HEV constituent des sites d'entrée très efficaces puisque l'on estime que dans l'organisme, à chaque seconde, plus de cinq millions de lymphocytes accèdent aux ganglions par cette voie [2].

\section{Organisation et rôle des vaisseaux HEV} Les cellules endothéliales extrêmement différenciées qui tapissent ces vaisseaux HEV sont caractérisées par une forme rebondie - dite cuboïdale - ainsi que par une forte expression d'enzymes (telles que la fucosyltransférase-VII [FucTVII], la $L$-selectin ligand sulfotransferase [LSST]) dédiées à la synthèse de molécules indispensables à la traversée vasculaire des lymphocytes (telles que les sialomucines membranaires hypersulfatées décorées par l'antigène reconnu par l'anticorps spécifique MECA-79) [2]. Des interactions moléculaires transitoires et successives entre ces sialomucines «MECA-79 positives » et la sélectine-L (ou CD62L) des lymphocytes circulants vont permettre aux lymphocytes de s'extraire du flux sanguin et de rouler à la surface de l'endothélium [1]. Ils termineront alors leur cheminement dans le ganglion lymphatique en adhérant fermement à la paroi vasculaire, puis en la traversant [2].

Ces dernières années, de nombreuses études ont montré que les vaisseaux HEV sont étroitement contrôlés par le microenvironnement tissulaire [3-5]. $\varepsilon n$ effet, à la naissance, ces vaisseaux présentent un phénotype immature (expression de la mucosal addressin cell adhesion molecule 1 [MAdCAM-1] au lieu de MECA-79) et n'acquièrent le phénotype HEV mature que durant le premier mois de vie postnatale [3]. Par la suite, chez l'adulte, les vaisseaux $H \varepsilon V$ restent extrêmement plastiques. Le clampage des vaisseaux lymphatiques afférents [4] d'un ganglion lymphatique provoque dans les cellules endothéliales la perte du phénotype HEV et leur transformation en cellules endothéliales classiques, tout comme le fait la culture de ces cellules isolées de leur tissu d'origine [5]. Enfin, ce phénotype $H \varepsilon V$ qui, en conditions physiologiques, est restreint exclusivement aux ganglions lymphatiques, peut aussi être induit au sein de tissus non lymphoïdes au cours de maladies inflammatoires chroniques (maladie de Crohn, polyarthrite rhumatoïde, thyroïdites) [2] et de cancer (sein, mélanome) [6]. La présence de vaisseaux HEV dans un tissu est toujours associée à un infiltrat massif de lymphocytes, néfaste dans le cas des maladies inflammatoires chroniques (entretient la boucle inflammatoire), mais de très bon pronostic dans le cas des tumeurs solides (favorise la réponse immunitaire antitumorale). II paraît donc essentiel de comprendre les mécanismes qui régissent l'induction et le maintien des vaisseaux HEV afin d'envisager de nouvelles pistes de traitements pour ces pathologies.

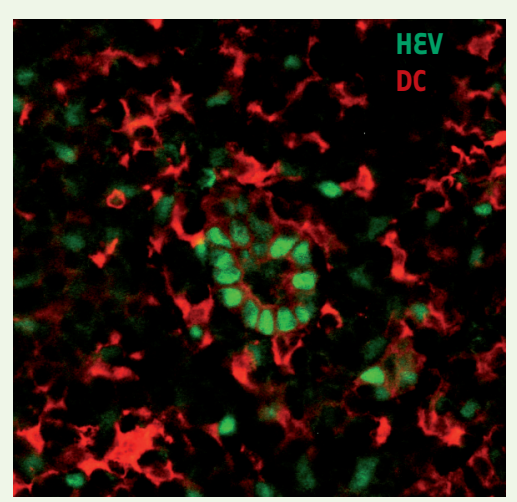

Figure 1. Interactions entre les cellules dendritiques ( $D C$, en rouge) et les cellules endothéliales d'un vaisseau HEV (en vert) au sein d'un organe lymphoïde secondaire. 
Rôle des cellules dendritiques (DC) dans le maintien des vaisseaux HEV Bien qu'il soit admis que le phénotype HEV est sous le contrôle du microenvironnement tissulaire, rien n'est connu de la nature des cellules impliquées. Du fait de la proximité étroite des $D C$ et des vaisseaux HEV dans les ganglions lymphatiques (Figure 1), nous avons fait l'hypothèse que les $D C$ pouvaient être

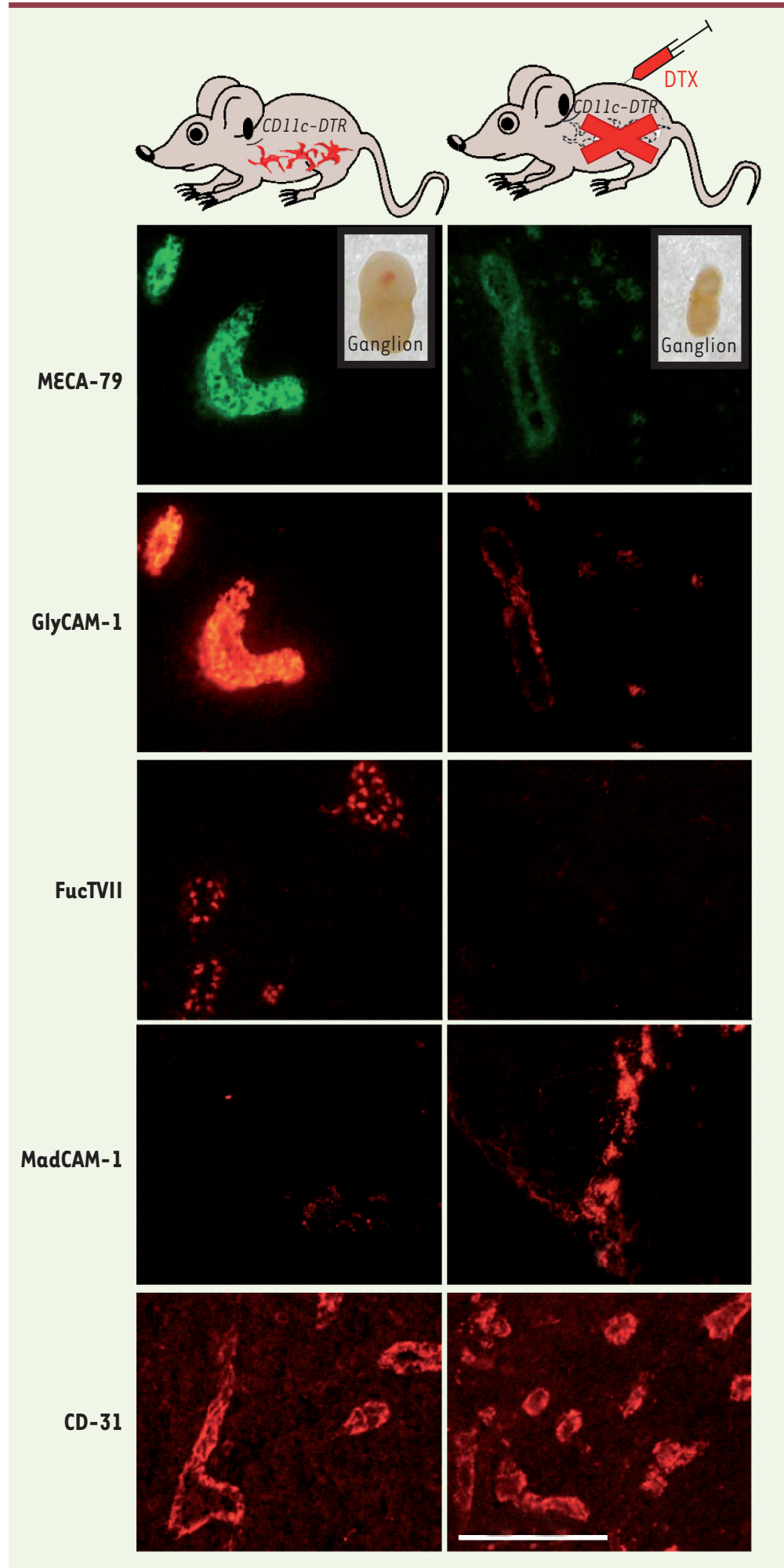

impliquées dans le contrôle du phénotype HEV [7]. Les DC sont les cellules présentatrices d'antigènes «professionnelles » de l'organisme découvertes en 1973 par Ralph Steinman (lauréat du Prix Nobel de médecine 2011) [8, 11]. Leur forme est étoilée avec de longs prolongements cellulaires leur permettant de capter les éléments de leur environnement dans les tissus périphériques, mais aussi de migrer vers les ganglions lymphatiques afin d'apprêter et de présenter les antigènes protéiques aux lymphocytes $T$ naïfs. En fonction de la situation, elles peuvent induire une tolérance vis-à-vis des antigènes du soi ou, inversement, une immunité adaptative protectrice contre des agents infectieux en délivrant des signaux d'activation aux lymphocytes $T$. Afin de tester l'hypothèse d'un rôle des $D C$ dans le maintien du phénotype HEV chez l'adulte sain, nous avons utilisé les souris transgéniques CDllc-DTR créées par S. Jung en 2002 [9]. Ce modèle murin exprime le récepteur simien de la toxine diphtérique (DTX) sous le contrôle du promoteur $\mathrm{CD} 1 \mathrm{lc}$ classiquement décrit comme spécifique des DC chez la souris. La DTX est une puissante toxine bactérienne pour la cellule dès lors qu'elle se lie à son récepteur membranaire. L'injection systémique de DTX à ces animaux permet la déplétion spécifique des $D C$ exprimant fortement CDllc des ganglions lymphatiques. Nous avons alors étudié le devenir des vaisseaux HEV une fois les $D C$ ganglionnaires éliminées par la DTX (Figure 2). L'analyse immunohistochimique des ganglions lymphatiques

Figure 2. Les cellules dendritiques (DC) maintiennent le phénotype mature et fonctionnel des cellules endothéliales HEV. La déplétion des DC par injection de DTX aux souris transgéniques CDIlc-DTR induit une dédifférenciation des cellules endothéliales qui conservent le marqueur vasculaire CD31 mais perdent les marqueurs HEV (MECA-79, GlyCAM-1 et FucTVII) pour adopter un phénotype immature «MAdCAM-1 positif » semblable à celui des cellules de la période postnatale. Les vaisseaux ne sont plus capables de recruter les lymphocytes circulant dans le sang, et les ganglions se vident de leur contenu cellulaire (encarts des photographies MECA-79). 
a révélé une perte des marqueurs HEV (MECA-79, GlyCAM-1, FucTVII) malgré une conservation des marqueurs endothéliaux classiques (CD31 ou platelet endothelial cell adhesion molecule [PECAM-1]). II existe une véritable dédifférenciation des vaisseaux HEV qui adoptent un phénotype immature similaire à celui qui est observé à la naissance et deviennent alors incapables de recruter les lymphocytes circulant dans le sang [7]. Les ganglions se vident presque entièrement de leur contenu cellulaire. L'analyse du recrutement par microscopie intravitale révèle un roulement trop rapide des lymphocytes sur l'endothélium qui ne peuvent plus s'arrêter et effectuer la diapedèse. L'ensemble des effets observés est réversible à l'arrêt des injections de DTX, mais aussi en réponse à l'injection sous-cutanée de grandes quantités de DC cultivées in vitro à partir de précurseurs de moelle osseuse.

Des expériences de cocultures entre des $D C$ et des cellules endothéliales $\mathrm{HEV}$ purifiées révèlent une interaction directe entre les deux partenaires par l'intermédiaire de la lymphotoxine (LT) $\alpha 1 \beta 2$ (ligand membranaire exprimé par les DC) et de son récepteur LT $\beta R$ (exprimé par l'endothélium) [10]. Cela a été démontré par l'utilisation du récepteur soluble LT $\beta R$-Ig bloquant cette signalisation lors des expériences de cocultures [7]. L'implication de la LT exprimée par les $D C$ dans le contrôle du phénotype HEV a été confirmée in vivo grâce à des expériences de greffes hématopoïétiques utilisant la moelle osseuse de souris déficientes pour la LT $\alpha$ (ou tumor necrosis factor $\beta$ [TNF $\beta]$ ).

\section{Interaction DC/HEV,}

une cible potentielle dans

I'inflammation et le cancer?

Les DC régulent l'accès des lymphocytes naïfs aux ganglions lymphatiques en contrôlant le phénotype des cellules endothéliales HEV. Les DC sont donc les véritables chefs d'orchestre de la surveillance immunitaire, non seulement parce qu'elles présentent les antigènes tissulaires aux lymphocytes T, mais aussi parce qu'elles en contrôlent l'étape préalable, l'accès des lymphocytes naïfs aux territoires lymphoïdes. Le couple DC/ $\mathrm{HEV}$ décrit dans notre étude constituerait ainsi une véritable unité fonctionnelle régulant l'accès d'un territoire à des cellules immunitaires. Le principal enjeu est maintenant de savoir si les DC sont aussi impliquées dans l'induction ectopique de vaisseaux $\mathrm{HEV}$ en conditions pathologiques et, si tel est le cas, quels en sont les mécanismes. On pourrait imaginer alors détourner à des fins thérapeutiques les mécanismes de contrôle pour freiner l'apparition de ces vaisseaux lors de pathologies inflammatoires chroniques (pour ralentir l'inflammation) ou, à l'inverse, favoriser leur développement lors des cancers (pour stimuler la réponse immunitaire antitumorale). $\diamond$

High endothelial venules (HEVs) and dendritic cells: a key functional unit for lymphocyte migration

\section{CONFLITS D'INTÉRÊTS}

Les auteurs déclarent n'avoir aucun conflit d'intérêts concernant les données publiées dans cet article.

\section{RÉFÉRENCES}

1. Von Andrian UH, Mempel TR. Homing and cellular traffic in lymph nodes. Nat Rev Immunol 2003 ; 3 : 867-78.

2. Girard JP, Springer TA. High endothelial venules (HEVs): specialized endothelium for lymphocyte migration. Immunol Today $1995 ; 16$ : 449-57.

3. Mebius RE, Streeter PR, Michie $S$, et al. A developmental switch in lymphocyte homing receptor and endothelial vascular addressin expression regulates lymphocyte homing and permits $\mathrm{CD4}^{+} \mathrm{CD}^{-}$cells to colonize lymph nodes. Proc Natl Acad Sci USA 1996 ; 93 : 11019-24.

4. Mebius RE, Streeter PR, Breve J, et al. The influence of afferent lymphatic vessel interruption on vascular addressin expression. J Cell Biol 1991 ; $115: 85-95$.

5. Lacorre DA, Baekkevold ES, Garrido I, et al. Plasticity of endothelial cells: rapid dedifferentiation of freshly isolated high endothelial venule endothelial cells outside the lymphoid tissue microenvironment. Blood $2004 ; 103: 4164-72$.

6. Martinet L, Garrido I, Filleron T, et al. Human solid tumors contain high endothelial venules: association with T- and B-lymphocyte infiltration and favorable prognosis in breast cancer. Cancer Res $2011 ; 71$ : 5678-87.

7. Moussion C, Girard JP. Dendritic cells control lymphocyte entry to lymph nodes through high endothelial venules. Nature $2011 ; 479$ : 542-6.

8. Steinman RM, Cohn ZA. Identification of a novel cell type in peripheral lymphoid organs of mice. I. Morphology, quantitation, tissue distribution. J Exp Med 1973; 137 : 1142-62.

9. Jung $S$, Unutmaz $D$, Wong $P$, et al. In vivo depletion of $\mathrm{CD} 1 \mathrm{lc}^{+}$dendritic cells abrogates priming of $\mathrm{CD} 8^{+}$ T cells by exogenous cell-associated antigens. Immunity 2002 ; 17 : 211-20.

10. Browning JL, Allaire N, Ngam- $\varepsilon k A$, et al. Lymphotoxinbeta receptor signaling is required for the homeostatic control of HEV differentiation and function. Immunity $2005 ; 23: 539-50$.

11. Zitvogel L, Amigorena S, Teillaud JL. Le printemps de l'immunité couronné à Stockholm. Prix Nobel de médecine 2011, Ralph M. Steinman. Med Sci (Paris) $2011 ; 27: 1028-34$.

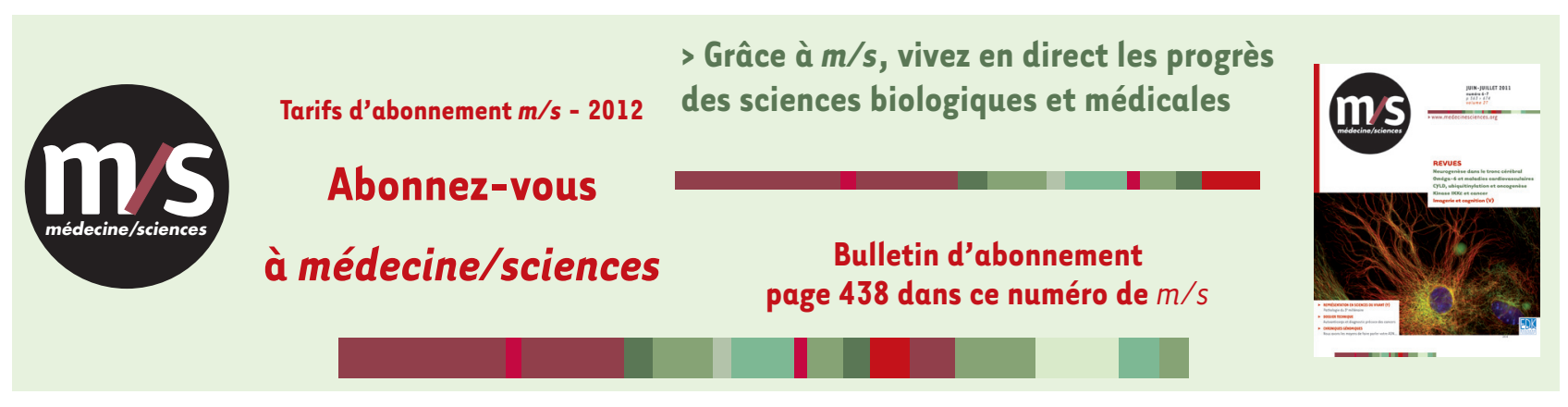

